\title{
Industrial Energy Load Profile Forecasting under Enhanced Time of Use Tariff (ETOU) using Artificial Neural Network
}

\author{
Mohamad Fani Sulaima ${ }^{1}$, Siti Aishah Abu Hanipah², Nur Rafiqah Abdul Razif ${ }^{3}$ \\ Intan Azmira Wan Abdul Razak ${ }^{4}$, Aida Fazliana Abdul Kadir ${ }^{5}$, Zul Hasrizal Bohari ${ }^{6}$ \\ Faculty of Electrical Engineering \\ Universiti Teknikal Malaysia Melaka \\ Melaka, Malaysia
}

\begin{abstract}
The demand response program involves consumers to mitigate peak demand and reducing global $\mathrm{CO} 2$ emission. In sustaining this effort, energy provider such as Tenaga Nasional Berhad (TNB) in Peninsular Malaysia has introduced Enhance Time of Use (ETOU) tariff. However, since 2015, small numbers join the ETOU program due to less confidence in managing their energy consumption profile. Thus, this study provides an optimum forecasting load profile model for TOU and ETOU tariffs using Artificial Neural Network (ANN). An industry's average energy profile has been used as a case study, while the forecasting technique has been conducted to find the optimum energy load profile congruently. The load shifting technique has been adopted under ETOU tariff price while integrating to the ANN procedure. A significant comparison in terms of cost reduction between TOU and ETOU electricity tariffs has been made. In contrast, ANN performance results in searching for the best-shifted load profile have been analyzed accordingly. From the proposed method, the total electricity cost saving has been founded to be saved for about $\mathbf{7 . 9 \%}$ monthly. It is hoped that this work will benefit the energy authority and consumers in future action, respectively.
\end{abstract}

Keywords-Time of use; artificial neural network; energy forecasting; load profile

\section{INTRODUCTION}

Demand Side Management (DSM) consists of Demand Response (DR) program to promote a better independent load management strategy for the consumers in dealing with the pricing and the time allocation. Under the DR framework, there is a price-based program related to time-based price. Under this structure, the consumers will select a program correlated to their energy load profile management. At the same time, they can shift the most available load to the lower price rate at a particular time zone [1]. One of the commands price-based programs is the Time of Use (TOU) tariff.

The TOU tariff has been proposed with various designs and depending on the national policy implemented through energy providers' tariffs to the consumers. The authors in [2] have highlighted the TOU time zones by the region. For example, India's command design was four zones, and China was fivezones and Brazil with three zones. However, the reference [3] presents a different opinion where the arrangement of the time zones design in TOU should be flexible enough to comply with two parties' needs: generation and consumer sides, respectively.

The TOU method design considering both consumers and generation was presented in [4], [5]. The founding is reliable, where the price elasticity consideration has been given attention. The marginal cost of the generation comes to the minimum profit rate is secured. Simultaneously, the promotion of the consumers' participants to the TOU program was a critical factor that was always being discussed. The TOU tariff design is also close to the residential consumers where the behavior and the appliances' arrangement to involve in load management is the command issue. Optimal load arrangement has been practiced while reflecting the energy provider or retailers' TOU tariff as explained in [6]. The consumers' response is essential for the TOU tariff's sustainable design, where the constitution of the demand response program toward human factor contribution is crucially needed.

In Peninsular Malaysia, is facing dramatic changes in the Malaysia Energy Supply Industry (MESI), electricity companies need to examine associated business models and a host of potential strategies to solve equations for over electricity demand from consumers especially in next MESI 2.0 [7]. TNB introduced many schemes, including ETOU tariffs, to benefit the commercial and industrial consumers, but less of them could implement load shifting and join the program correctly.

Thus, in this paper, the analysis and suggestion for the ETOU tariff study to deal with industrial load profile is explored. Meanwhile the implementation of the artificial algorithm has been given attention to produce the optimal profile of the energy to meet the lower cost of the electricity under ETOU program. The related works have been presented in Section II. Meanwhile, the detailed explanation of the specific industrial load profile formulation under the ETOU tariff price has been written in Section III. Meanwhile, Section IV introduced ANN's implementation in processing the load profile forecasting under the ETOU tariff price. The simulation results comparing the actual load profile and the optimization load profile are demonstrated in Section V. The last Section VI concludes the overall study contribution and recommendation for an available future research opportunity. 


\section{RELATED WORKS}

Unsuitable tariffs for different load profiles will increase energy costs and lead to the DR program's wrong perception [8]. In [9], [10], the ETOU tariff price has been adopted to the commercial and industrial consumers' reference energy profile. The finding has been analyzed where the flexibility of the load shifting weightage has been constructed accordingly. There were limitations found that the minimum load shifting weightage was higher for the consumers to gain the cost reduction.

The previous study's suggestion contributes to implementing the optimization algorithms to find the optimal load profile that reflects the ETOU tariff price focusing on the Malaysian electricity market condition. The application of the Particle Swarm Optimization (PSO) algorithm has been presented in reference [11] to find the appropriate load categories under six groups of the sectors in peninsular Malaysia. The powerful load management strategies to reduce total electricity under ETOU tariff have been decreased by using Ant Colony Optimization (ACO) [12] and [13]. The authors in [2] present an Evolutionary Algorithm (EA) as the optimal search of load profile in tackling the ETOU price. The future recommendation has been made in the field of knowledge where the optimization algorithm's impact would be further studied to load profile forecasting under the ETOU tariff price.

The application of a conventional vector machine in determining the load forecasting was critically discussed in [14]. Combining the optimization algorithm produced more impact in reducing the root means square value while updating the convergence time, as proven by [15]. As different studies reported of the application of ANN-Self Organizing Mapping for the load forecasting, the significant analysis made has contributed to the excellent summary of the group clustering for the electricity profiles as the example in [16]-[18]. However, those references less reflect the tariff structure in considering the TOU's impact on benefiting consumers of the electricity cost reduction concurrently.

Regarding the load profile forecasting, which reflects ETOU electricity tariff in peninsular Malaysia, there is no particular study to date for the best of the knowledge. Thus, in this study, the mathematical equation stage for ETOU price has been explored and explained. In contrast, the effective formulation for optimal load profile forecasting and the ANN's implementation has been proposed congruently. The significant case study of an industry load profile from the medium voltage category was chosen with the operation time was 24 hours. In contrast, the optimal load profile results to reduce the electricity cost have been explained accordingly.

\section{FORMULATION OF INDUSTRIAL ENERGY LOAD FORECASTING UNDER ETOU TARIFF PRICE}

The flow of the ETOU mathematical stage for demand pricing is presented as follows: Based on the average load profile data, the range for peak time, off-peak time, and maximum demand are identified. Maximum demand cost, $\mathrm{C}_{\mathrm{TMD}}$ :

$C_{T M D}=P_{M D} \times R_{T M D} ;(M Y R)$
The average load profile versus time (energy consumption) for every peak are plotted. The reference formula was obtained from the graph by performing regression, and data analysis processes have been shown in the results. Remodel the reference formula by substituting the total peak cost and rated peak value to find a new peak and off-peak ratio. Thus, the total off-peak cost is written as $\mathrm{Y}_{\mathrm{TOP}}$ :

$Y_{T O P}=P_{T O P} \times R_{T O P} ;(M Y R)$

Total peak cost, $\mathrm{Y}_{\mathrm{TP}}$ :

$Y_{T P}=P_{T P} \times R_{T P} ;(M Y R)$

The formulas for the total cost for every peak are designed by using new ratio values. Verify the total formula cost for peak and off-peak by plotting new graph time versus power using every peak formula's total cost. Perform data analysis and regression to get the formula from the graph. Compared the ratio value with the calculated ratio value from (4). The total cost for TOU tariff, $\mathrm{T}_{\mathrm{T}}$ is:

$T_{T}=C_{T O P} \times C_{T P} \times C_{T M D} ;(M Y R)$

Where;

$\mathrm{C}_{\mathrm{TMD}}=$ total electricity cost for maximum demand

$\mathrm{C}_{\mathrm{TOP}}=$ total electricity cost for off-peak time

$\mathrm{C}_{\mathrm{TP}}=$ total electricity cost for peak time

Repeat all Eq. (1) until (4) for ETOU tariff, but the arrangement of the mid-peak cost follows Eq. (3). Thus, the total cost for ETOU tariff, $\mathrm{T}_{\mathrm{E}}$ is:

$T_{E}=C_{E O P} \times C_{E M P} \times C_{E P} \times C_{T M D} ;(M Y R)$

Where;

$\mathrm{C}_{\mathrm{TMD}}=$ total electricity cost for maximum demand

$\mathrm{C}_{\mathrm{EOP}}=$ total electricity cost for off-peak time

$\mathrm{C}_{\mathrm{EMP}}=$ total electricity cost for medium peak time

$\mathrm{C}_{\mathrm{EP}}=$ total electricity cost for peak time

The $\mathrm{T}_{\mathrm{T}}$ and $\mathrm{T}_{\mathrm{E}}$ formula is tested with a conventional method by substituting all the ratio, $\mathrm{Q}$ and tariff rates, $\mathrm{R}$ values, and formula value. To observed TOU and ETOU tariff, graph tariff versus cost TOU and ETOU are performed and analyzed.

Hence, the optimal formulation for daily energy consumption cost (EC) is Eq. (6).

$E C=\sum_{t=1}^{48} R(t) L(t)$

Where; $\mathrm{R}(\mathrm{t})=$ ETOU rate of each hour

$\mathrm{L}(\mathrm{t})=$ Load of each 30 minutes $(0<t \leq 48)$

Since the ETOU tariff price is fixed every day, consumers may shift their loads to reduce energy costs. Then the load shifting reflect the energy cost, $\mathrm{EC}_{\mathrm{R}}$ could be written as

$E C_{R}=\sum_{t=1}^{48} R(t) L_{R}(t)$

Where; $\mathrm{EC}_{\mathrm{R}}=$ Energy cost of that day under load shifting; 
$\mathrm{L}_{\mathrm{R}}(\mathrm{t})=$ Load shifting of each 30 minutes

$(0<t \leq 48)$;

The industrial consumers' objective function to enjoy minimum energy cost by load shifting is written in Eq. (8) accordingly.

$\min \left(E C_{R}\right)=\min \left[\sum_{t=1}^{48} R(t) L_{R}(t)\right]$

The total energy consumption in a day for baseload or actual load and after load shifting must be equal has been set as the constraint. The consumers may only change some of their loads from the high price periods to the low-price periods. But the total consumption maybe not changed due to similar behaviors in their daily life or production. However, in the condition of the simulation forecasting process, if the load shifting condition less than 5\% from the actual load, the results can also be considered for comparison. Thus, the constrain condition could be written as:

$\sum_{t=1}^{48} L_{R}(t) \approx \sum_{t=1}^{48} L(t)$

\section{ImPlementation OF ARTIFICIAL NEURAL NETWORK}

Table I shows stages that are involved in load forecasting and optimization by using ANN. Meanwhile, the explanation of the implementation has been written in sub-section A until E accordingly.

TABLE I. ANN PRocess to DETERMine the Optimal LOAD PROFILE UNDER ETOU TARIFF PRICE

\begin{tabular}{|l|l|}
\hline Stages & Process \\
\hline Step 1 & Data Organization \\
\hline Step 2 & Data Training \\
\hline Step 3 & Data testing \\
\hline Step 4 & Data Forecasting \\
\hline
\end{tabular}

A. Data Organization

The previous energy profile data is used as input data. The data include daily energy profile value in minutes in January 2016. Twenty-eight data sets are divided into two main groups, which are x_(LoadProfile) and y_EnergyCost. An $\mathrm{x}$ _(LoadProfile) is industry energy profile for 14 data set for working days. Meanwhile, y_EnergyCost is for total energy cost per day with ETOU tariff for 14 data set for working days.

\section{B. Data Training}

In the training process, the data structure must be normalized. The normalization is copied to the map structure during the trained ANN. The data input and output will normalize and scale the variable values between negative and positive ones. The optimum number of neurons must be considered during normalization. The data are normalized by using the following formula:

$x_{n}=\left[x_{j}-\left(\frac{x_{\max }+x_{\min }}{2}\right)\right] \div\left(\frac{x_{\max }-x_{\min }}{2}\right)$
Where; $\mathrm{X}_{\mathrm{n}} \quad$ = Normalized data;

$\mathrm{X}_{\mathrm{j}} \quad=$ Actual data;

$\mathrm{X}_{\max }=$ Maximum value in actual data;

$\mathrm{X}_{\min }=$ Minimum value in actual data;

\section{Data Testing}

After training, the maps are tested with testing data of energy profile and energy cost. This method is to associate the energy profile and tariff simultaneously. The data testing process will obtain the pattern for the most suitable load shifting profile.

\section{Data Forecasting}

After testing, all the load data will be forecasted. The curve of energy profile forecasting and actual profile is obtained by the simulation process accordingly.

\section{RESUlts AND ANALYSIS}

Fig. 1 shows the energy profile, which is having different power consumes during every time zone. The industrial electricity installation is registered under E2 tariff while comparison has been made to E1 flat and E1 ETOU tariff price. The energy pattern for January 25, 2016 (ThursdayWorking day) showed that the energy was fully consumed during peak time zones. The Red dot on the figure indicates that the max power consumes $3,330 \mathrm{~kW}$, which will be considered maximum demand. The maximum demand position is at 3:00 PM, which is in the peak time zone for TOU and ETOU tariffs. Fig. 2 shows the energy consumed profile for an industrial within 14 days (working day). The profiles demonstrate power consumption for working days is just the same, which is fully consumed during normal working hours, and only $50 \%$ operate at night. For the normal 24 hours operation in the manufacturing batch process, the repeating profile would be expected. The load profile index would be calculated as well to see the level of the significant correlation of the maximum demand and the energy consumption.

The best prediction of the minimum energy cost pattern was produced in Fig. 3. The blue line refers to the actual energy cost with ETOU tariff, while the red line shows the new energy cost after optimization with a regression of 0.7003 . The graph prediction graph showed various changing patterns to reach the minimum energy cost. Since the ETOU tariff has been divided by Six-Segmentation of the zone that reflect three prices unit, the investigation could be analyzed based on that points. The simulation power profile for the ETOU tariff on the mid-peak and peak zones have followed the baseline profile. However, the consumption of the electricity has reduced significantly. The power consumption on off-peak zone from the $10: 00 \mathrm{PM}$ to the $8: 00 \mathrm{AM}$ has increased tremendously to show the performance of the algorithm to find the optimal load to be shifted from peak to off-peak zone. Thus, all those condition during the adaptation of the ANN has contributed to the cost profile such presented in Fig. 3 accordingly. 


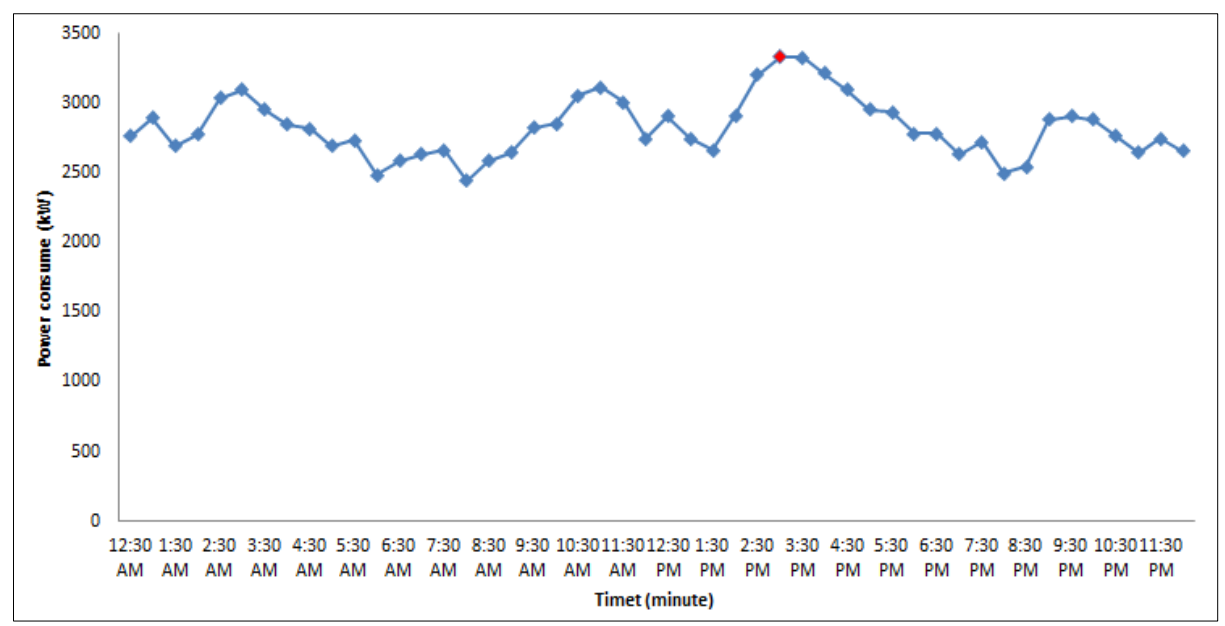

Fig. 1. Energy Profile in every 30 minutes for 24 hours from 12:30 am until 12:00am (average).

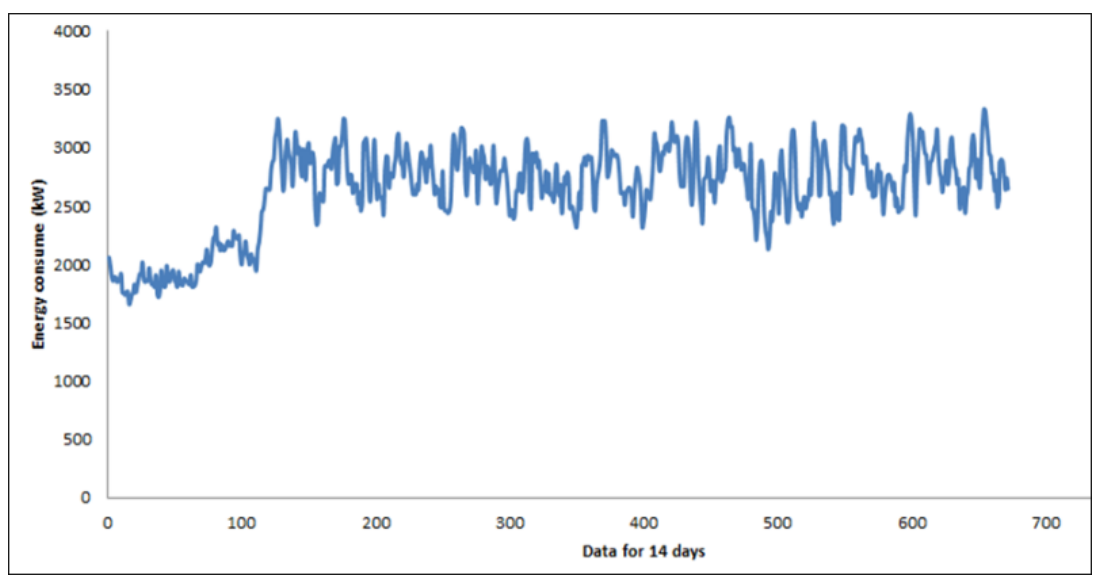

Fig. 2. The Energy Pattern of Industrial Sector for 14 days.

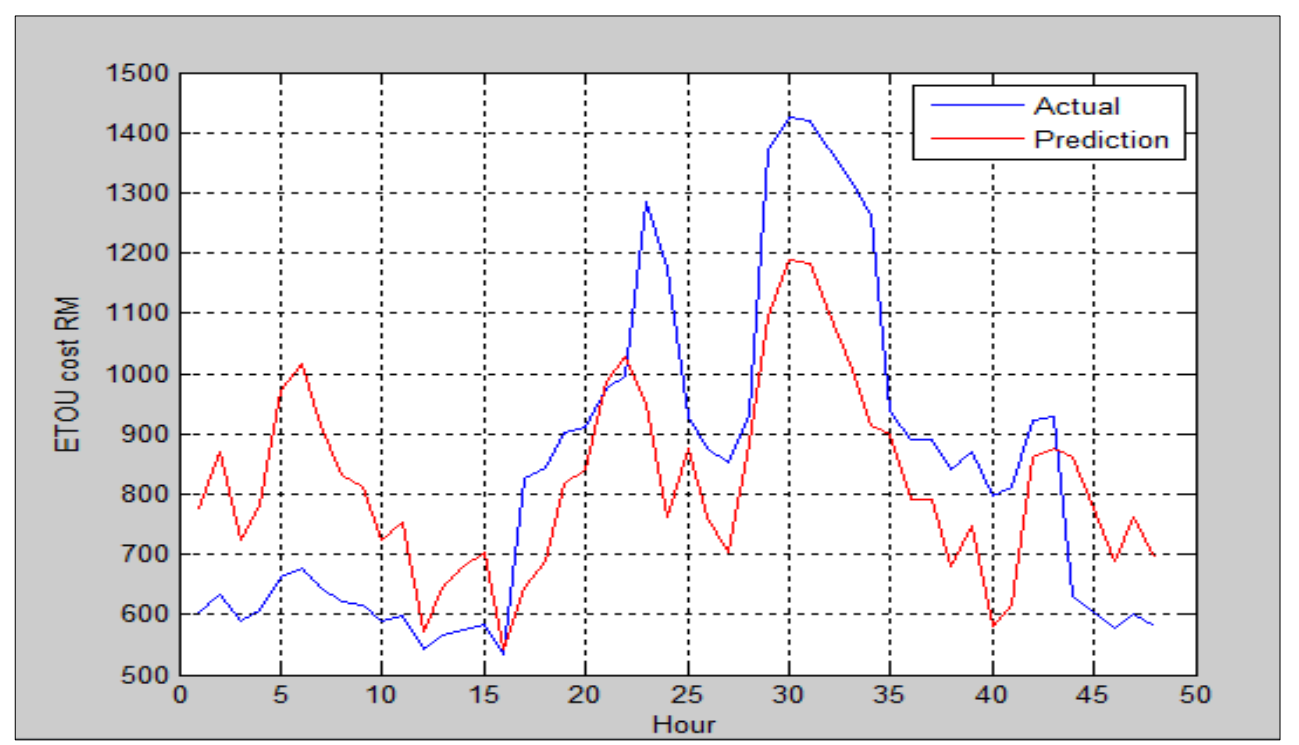

Fig. 3. The Energy Cost Pattern for Actual Data and Prediction Data. 


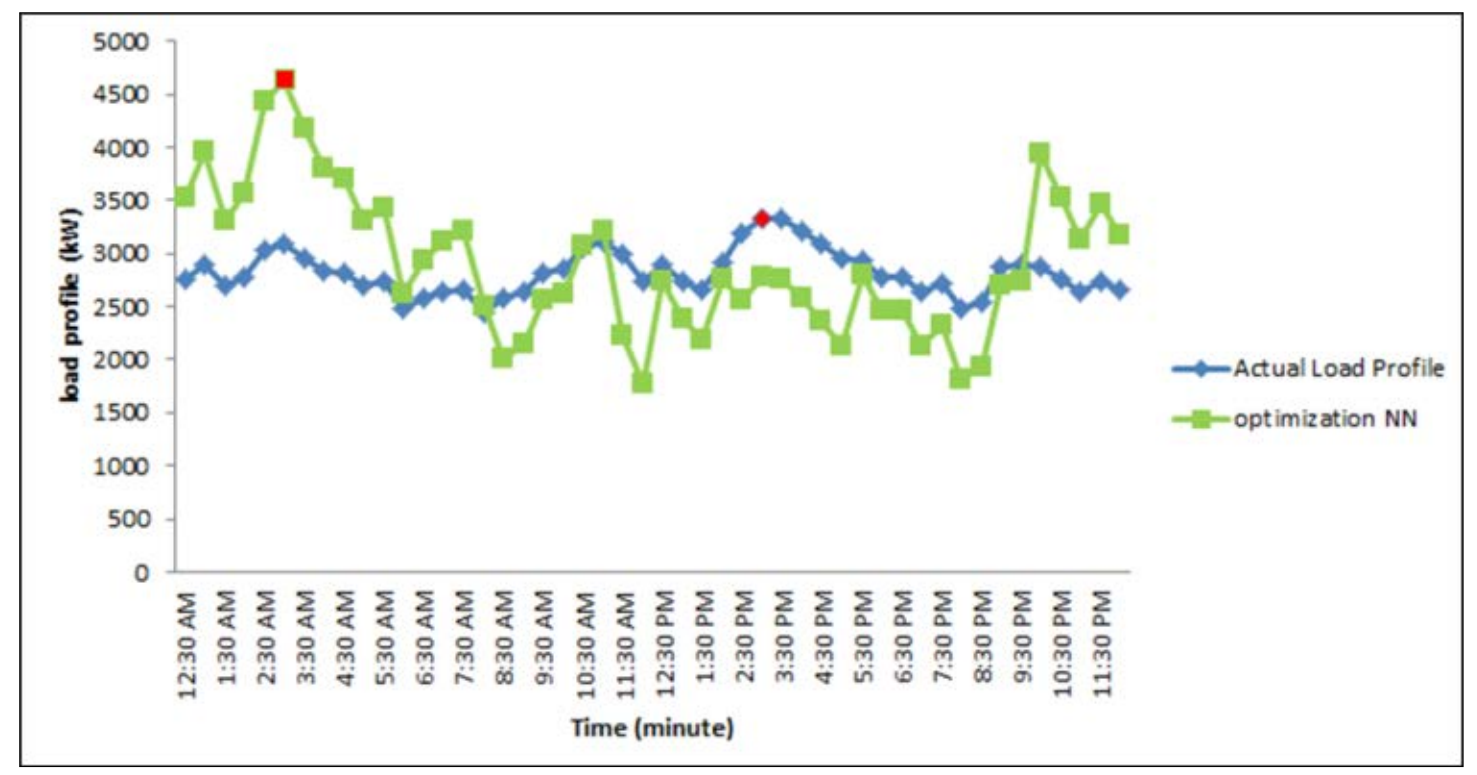

Fig. 4. The Energy Profile Pattern for Actual Data and Optimization Data.

The optimal load response of typical industrial load to ETOU power price for demand-side management are shown in Fig. 4. The customer shifts some of the loads from the high price period to the low-price period in order to achieve minimum energy cost in the day. The load is reduced about $10 \%$ at the price peak (about 3:00 PM), and 15\% reduction at mid-peak (about 11:30 AM). Meanwhile, the load is increased by about $20 \%$ at the price low peak (about 3:00 AM) and 15\% improved at low peak (about 10:00PM). Overall load optimization in average ratio was 40:60 of peak and off-peak zone, respectively.

The maximum demand position also shifted from peak region to mid peak region while the peak demand was allocated in off-peak concurrently. It is indicated that the peak electricity consumption is reduced, and the off-peak electricity consumption increases significantly, which affects the power system's regular operation. Table II shows the reduction of the analysis for the energy and maximum demand cost based on two types of tariffs offered to the consumer. Since the ETOU offers off-peak price for the weekend, the advantage has gained to the industrial profile when running the same operation every day. Total forecasting monthly electricity bill when using the optimization method was reduced up to $7.9 \%$ which approximately MYR $103,453.00$ or yearly saving for about MYR 1,241,436.00.

Besides, Table III presents the comparison of the cost reduction between the case of optimum TOU tariff and case of the optimum ETOU tariff price accordingly. Both of the price rates has been used in the simulation. Meanwhile, the optimum load profile was defined as explained before. Through the comparison, both TOU and ETOU are produced excellence cost reduction for approximately $5 \%$ $8 \%$, but it would be recommended that the ETOU tariff scheme be able to benefit the consumers with the condition that the optimum load must be applied. The study's cost-saving recorded shows the significant contribution of the demand response program to the consumers. In contrast, the peak demand would be projected to be shifted to off-peak hour. Thus, the peak demand movement condition contributes to effort the efficiency management of the generation side where the critical type of the generation would not be run in the long time every day.

TABLE II. The Cost COMPARISON BETWEen ACtual ENERgy PRofile AND OPTIMIZATION ENERgy PROFILE

\begin{tabular}{|l|l|l|l|}
\hline Records & $\begin{array}{l}\text { Actual Case } \\
\text { E1 flat }\end{array}$ & $\begin{array}{l}\text { Optimization } \\
\text { Case E1 ETOU }\end{array}$ & Reduction \\
\hline Daily Energy cost & $40,201.00$ & $39,369.52$ & $2.07 \%$ \\
\hline $\begin{array}{l}\text { Maximum demand } \\
(\mathrm{kW})\end{array}$ & 3,330 (peak) & 3,210 (mid peak) & $3.60 \%$ \\
\hline $\begin{array}{l}\text { Maximum demand cost } \\
\text { (MYR) }\end{array}$ & $98,568.00$ & $95,016.00$ & $3.60 \%$ \\
\hline $\begin{array}{l}\text { Total Monthly } \\
\text { Electricity Cost (MYR) }\end{array}$ & $1,304,598.00$ & $1,201,145.44$ & $7.90 \%$ \\
\hline
\end{tabular}

TABLE III. THE COMPARISON OF ENERGY COST BY USING OPTIMIZATION ENERGY PROFILE

\begin{tabular}{|l|l|l|c|}
\hline Tariff & $\begin{array}{l}\text { Without Optimization } \\
\text { Monthly Energy Cost, } \\
\text { (MYR) }\end{array}$ & $\begin{array}{l}\text { With Optimization } \\
\text { Monthly Energy } \\
\text { Cost, (MYR) }\end{array}$ & Saving (\%) \\
\hline TOU & $1,262,019.00$ & $1,194,424.80$ & 5.36 \\
\hline ETOU & $1,318,238.00$ & $1,201,145.44$ & 8.80 \\
\hline
\end{tabular}

\section{CONCLUSION}

In this study, the ANN has been applied to forecast the optimum load profile under TOU and ETOU tariff prices. The comparison of the both tariffs has been done where the benefit of the TOU and ETOU price could be received by consumers were quantified based on the monthly and yearly results. Meanwhile, the significant is shifted load must converge in the zone of the mid-peak for the lower charge of the maximum demand. As the investigation statement presents that the ETOU can be optimum adopted by the consumers where the minimum 
load shifting condition is applied. Besides, the electricity bill's cost can be reduced; the peak demand has been shifted to the off-peak zone that brings benefits to the generation side. The future recommendation of the research continuity can be explored on the comparison of the forecasting algorithms by using the other techniques while using a multi-objective environment. Meanwhile other factor of the effect toward load factor index and the energy economic side should be considered as well.

\section{ACKNOWLEDGMENT}

The authors would like to thank Universiti Teknikal Malaysia Melaka (UTeM) for all the support given. The publication of the paper is sponsored under the publication support scheme under Centre for Research and Innovation UTeM (CRIM).

\section{REFERENCES}

[1] F. Meyabadi and M. H. Deihimi, "A review of demand-side management: Reconsidering theoretical framework,” Renew. Sustain. Energy Rev., vol. 80, no. January 2016, pp. 367-379, 2017.

[2] M. F. Sulaima, N. Y. Dahlan, Z. M. Yasin, N. A. M. Asari, and Z. H. Bohari, "Optimum enhance time of use (ETOU) for demand side electricity pricing in regulated market: An implementation using evolutionary algorithm,” Indones. J. Electr. Eng. Comput. Sci., vol. 8, no. 1, 2017.

[3] V. Venizelou, N. Philippou, M. Hadjipanayi, G. Makrides, V. Efthymiou, and G. E. Georghiou, "Development of a novel time-of-use tariff algorithm for residential prosumer price-based demand side management," Energy, vol. 142, pp. 633-646, 2018.

[4] M. Pauzi Abdullah, M. N. Nazatul Shiema, M. Y. Hassan, and F. Hussin, "Optimizing Time of Use Electricity Pricing in Regulalted Market," J. Teknol., vol. 7, pp. 31-39, 2013.

[5] N. S. M. Nazar, M. P. Abdullah, M. Y. Hassan, and F. Hussin, "Timebased electricity pricing for Demand Response implementation in monopolized electricity market,” in SCOReD 2012 - 2012 IEEE Student Conference on Research and Development, 2012, pp. 178-181.

[6] S. Yilmaz, S. Weber, and M. K. Patel, "Who is sensitive to DSM? Understanding the determinants of the shape of electricity load curves and demand shifting: Socio-demographic characteristics, appliance use and attitudes,” Energy Policy, vol. 133, pp. 1-13, 2019.
[7] Energy Commission (Malaysia), "Reimaging Malaysia Energy Supply Industry (MESI 2.0)," 2019.

[8] S. Mohajeryami, I. N. Moghaddam, M. Doostan, B. Vatani, and P. Schwarz, "A novel economic model for price-based demand response," Electr. Power Syst. Res., vol. 135, pp. 1-9, 2016.

[9] N. A. M. Azman, M. Pauzi Abdullah, M. Y. Hassan, and D. M. Said, "Enhanced Time of Use Electricity Pricing for Industrial Customers in Malaysia,” Indones. J. Electr. Eng. Comput. Sci., vol. 6, no. 1, pp. 155160, 2017.

[10] N. A. M. Azman, M. P. Abdullah, M. Y. Hassan, D. M. Said, and F. Hussin, "Enhanced time of use electricity pricing for commercial customers in Malaysia,” Pertanika J. Sci. Technol., vol. 25, pp. 285-294, 2017.

[11] M. F. Sulaima, N. Y. Dahlan, Z. M. Yasin, M. M. Rosli, Z. Omar, and M. Y. Hassan, "A review of electricity pricing in peninsular Malaysia: Empirical investigation about the appropriateness of Enhanced Time of Use (ETOU) electricity tariff,” Renew. Sustain. Energy Rev., vol. 110, pp. 348-367, 2019.

[12] M. Sulaima, N. Dahlan, and Z. Yasin, "Effective Electricity Cost Management in a Manufacturing Operation by Using Optimal ETOU Tariff Formulation,” Int. J. Electr. Electron. Syst. Res., vol. 15, pp. 8293, 2019.

[13] M. F. Sulaima, N. Y. Dahlan, M. H. Isa, M. N. Othman, Z. M. Yasin, and H. A. Kasdirin, "ETOU electricity tariff for manufacturing load shifting strategy using ACO algorithm,” Bull. Electr. Enginnering Informatics, vol. 8, no. 1, pp. 21-29, 2019.

[14] D. Niu, Y. Wang, and D. Dash, "Power load forecasting using support vector machine and ant colony optimization,” Expert Syst. Appl., vol. 37, pp. 2531-2539, 2010.

[15] A. Ghasemi, H. Shayeghi, M. Moradzadeh, and M. Nooshyar, "A novel hybrid algorithm for electricity price and load forecasting in smart grids with demand-side management," Appl. Energy, vol. 177, pp. 40-59, 2016.

[16] N. . Z. N. F. . G. N.N Atira; I. Azmiara, Z.H. Bohari, "Medium Term Load Forecasting Using Statistical Feature Self Organizing Maps ( SOM ),” J. Telecommun. Electron. Comput. Eng., vol. 11, no. 2, pp. 25-29, 2019.

[17] X. Lin, Z. Tian, Y. Lu, H. Zhang, and J. Niu, "Short-term forecast model of cooling load using load component disaggregation," Appl. Therm. Eng., vol. 157, pp. 1-13, 2019.

[18] Z. H. Bohari, H. S. Azemy, M. N. M. Nasir, M. F. Baharom, and M. F. Sulaima, "Reliable Short Term Load Forecasting Using Self Organizing Map (SOM) in Deregulated Electricity Market,” J. Theor. Appl. Inf. Technol., vol. 79, no. 3, pp. 389-394, 2015. 